\title{
PROSPECTS FOR A VERY HIGH POWER CW SRF LINAC*
}

\author{
R.A. RIMMER ${ }^{+}$
}

Thomas Jefferson National Accelerator Facility, 12000 Jefferson Avenue, Newport

News, VA 23606, USA

\begin{abstract}
Steady development in SRF accelerator technology combined with the success of large scale installations such as CEBAF at Jefferson Laboratory and the SNS Linac at ORNL gives credibility to the concept of very high average power CW machines for light sources or Proton drivers. Such machines would be powerful tools for discovery science in themselves but could also pave the way to reliable cost effective drivers for such applications as neutrino factories, an energy-frontier muon collider, nuclear waste transmutation or accelerator driven subcritical reactors for energy production. In contrast to machines such as ILC that need maximum accelerating gradient, the challenges in these machines are mainly in efficiency, reliability, beam stability, beam loss and of course cost. In this paper the present state of the art is briefly reviewed and options for a multi-GeV, multi-MW CW linac are discussed.
\end{abstract}

\section{Introduction}

Large-scale CW SRF installations such as CEBAF [1] and LEP [2] have demonstrated that CW SRF technology can work reliably for demanding applications and facilities with $24 / 7$ user operations, in parameter regimes where no other current technology would be viable. The recent success of the pulsed SNS at ORNL further supports the case, delivering high beam current at high duty factor to meet the milestone of $1 \mathrm{MW}$ beam power for users. Extrapolating this performance to a large CW machine presents the exciting prospect of an unprecedented source of high-energy protons for a wide range of applications. Such ideas are further stimulated by the proposal of a new multi-GeV high intensity proton driver at Fermilab, code

\footnotetext{
${ }^{*}$ This work supported by the U.S. Department of Energy, contract DE-AC05-84-ER40150.

† rarimmer@jlab.org
} 
named Project X. Initially planned as an $8 \mathrm{GeV}$ pulsed linac based on ILClike SRF technology, there now appears to be serious consideration of making some or even all of the machine operate $\mathrm{CW}$ to provide simultaneous high intensity beam to multiple experiments as well as continuing to support the very high energy users. Such a machine would be the backbone of the Fermilab program for decades, supporting a worldleading neutrino physics program and other landmark experiments and eventually enabling an energy frontier muon collider to be envisaged on the Fermilab site.

The good news is that many "proton driver optimized" cavities suitable for CW operation exist or are in development e.g. at ANL, Triumf, JLab (Figure 1) and KEK, as well as cryomodule designs that can accommodate them, so designers need not feel constrained by the narrow bounds of the TESLA-like technology

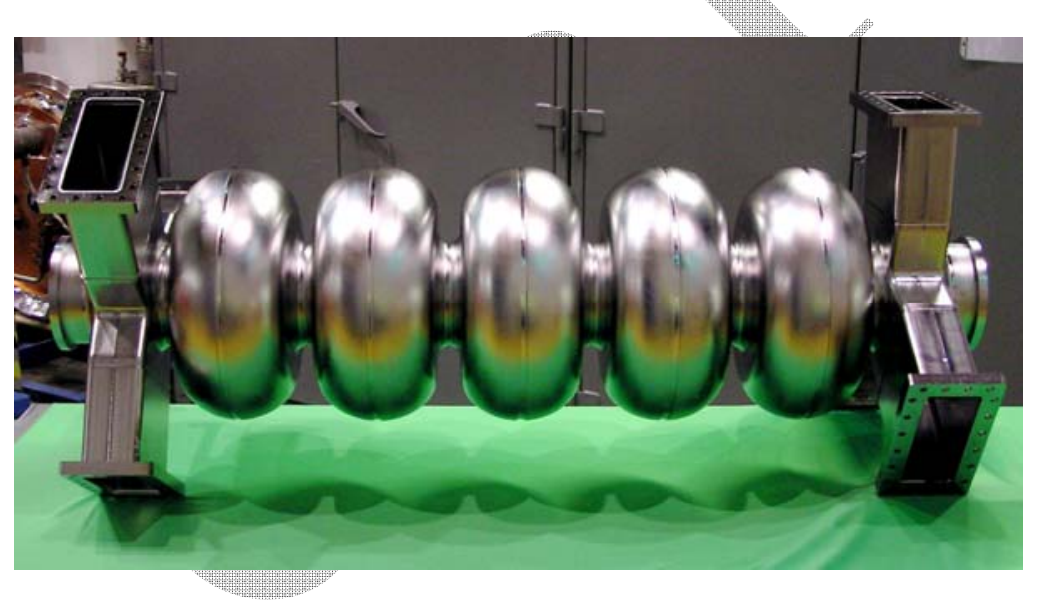

Figure 1. JLab Prototype $750 \mathrm{MHz}$ high-current cavity. This cell shape is optimized for beta=1 high current beams. Higher-order modes are tuned to safe frequencies to minimize power extraction from the beam. Waveguide HOM dampers provide very strong damping of unwanted modes.

SRF cavity performance also continues to advance, not only on the gradient frontier but also in terms of operating efficiency through improved 
geometry factor and $Q_{o}$ at typical CW operating gradients. In fact the same electropolishing (EP) technology responsible for gradient advancements for ILC has also been shown to give significant improvements in $Q_{o}$ at more modest fields.

Experience with operating machines means that the beam-break-up (BBU) limits are quite well understood and mitigations have been developed and demonstrated in the form of HOM damping or detuning (and feedback in the case of multi-pass or re-circulating machines). Average currents in the tens or even hundreds of $\mathrm{mA}$ are now quite possible in this regard. The main remaining challenge is halo control to minimize beam loss and activation.

Large $2 \mathrm{~K}$ cryogenic plants are also becoming more efficient, both in peak capacity and turn down mode, addressing one of the largest cost drivers in operation and construction of such machines.

Even CW RF sources continue to be developed with lower cost, greater efficiency and longer life. The widespread adoption of inductive output tubes (IOTs) by the broadcast industry provides a stable technology base for these devices, which can be adapted to a wide variety of frequencies and power levels. Of course klystrons remain viable and with multi-beam tubes, annular or sheet beams and other developments still in progress they may remain competitive long into the future. Magnetrons are one of the oldest sources of RF power and have good efficiency and low construction cost but being oscillators rather than amplifiers they have not been much favored for large accelerators (although a significant number of medical and industrial machines are powered by them). However advances in digital switching high-voltage power supplies and fast low level RF control do allow the prospect for modulation and phase stabilization of the magnetron output, and if this can be demonstrated to an acceptable level of stability they could become a very favorable option for high power $\mathrm{CW}$ machines.

The science case for new machines is very strong and there is encouraging progress in both the technology and the user community interest towards their realization.

\section{State of the Art}

Large installations such as CEBAF and SNS may be used as benchmarks to assess the state of readiness of a CW machine for applications such as ADS. Although CEBAF is a $1.5 \mathrm{GHz}$ electron accelerator it does operate CW with more than $1 \mathrm{GeV}$ of installed capability from forty cryomodules, Figure 2, 
(plus two and a quarter in the injector) and has been running for over a decade with high availability for up to three simultaneous user end stations. The average current is modest but because the machine is operated in a 5 pass recirculation mode the maximum energy is $6 \mathrm{GeV}$ (originally $4 \mathrm{GeV}$ ) and the beam power can reach $1 \mathrm{MW}$, the maximum administrative site limit. As CEBAF was first proposed more than 25 years ago the initial gradient specification was very conservative by present standards at 5 $\mathrm{MV} / \mathrm{m}$. In fact even during the initial production run many cavities comfortably exceeded this mark. At $4 \mathrm{~kW}$ it had what was at the time the worlds largest $2 \mathrm{~K}$ cryogenic plant. Recently ten of the original cryomodules have been refurbished with a goal of $12.5 \mathrm{MV} / \mathrm{m}$ ( $50 \mathrm{MV}$ per cryomodule), reusing the original cavities but with improved preparation techniques. The machine will shortly be upgraded to $12 \mathrm{GeV}$, Figure 3 , with the addition of ten new high performance cryomodules adding an additional $1 \mathrm{GeV}$ per pass, a fourth hall and an additional $4.5 \mathrm{~kW}$ central helium liquefier (CHL2). Some parameters for the upgrade cryomodules are listed in Table 1. These along with the demonstrated SNS performance may be useful benchmarks for new projects.

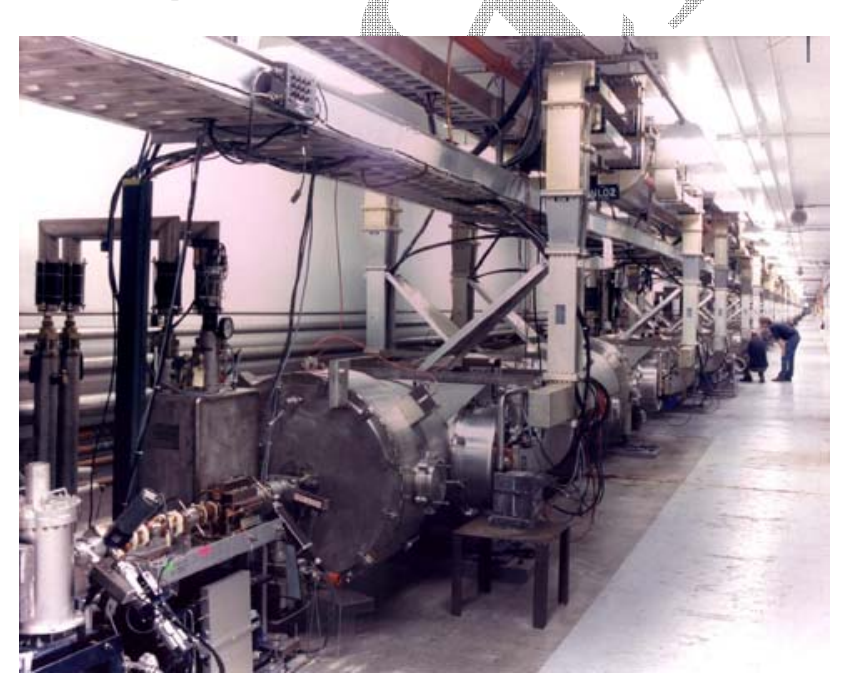

Figure 2. One of the two CEBAF linacs, each comprising 20 original cryomodules assembled inhouse, plus $2^{1 / 4}$ in the injector. 


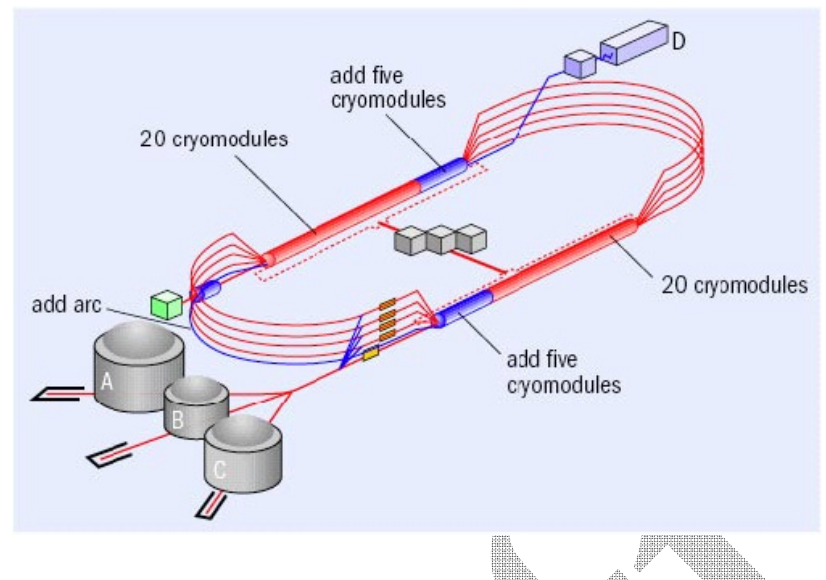

Figure 3. Upgrade of the CEBAF 5-pass CW recirculating linac from 6 to $12 \mathrm{GeV}$. Five new high performance cryomodules will be installed in each linac along with one extra arc and a new experimental hall.

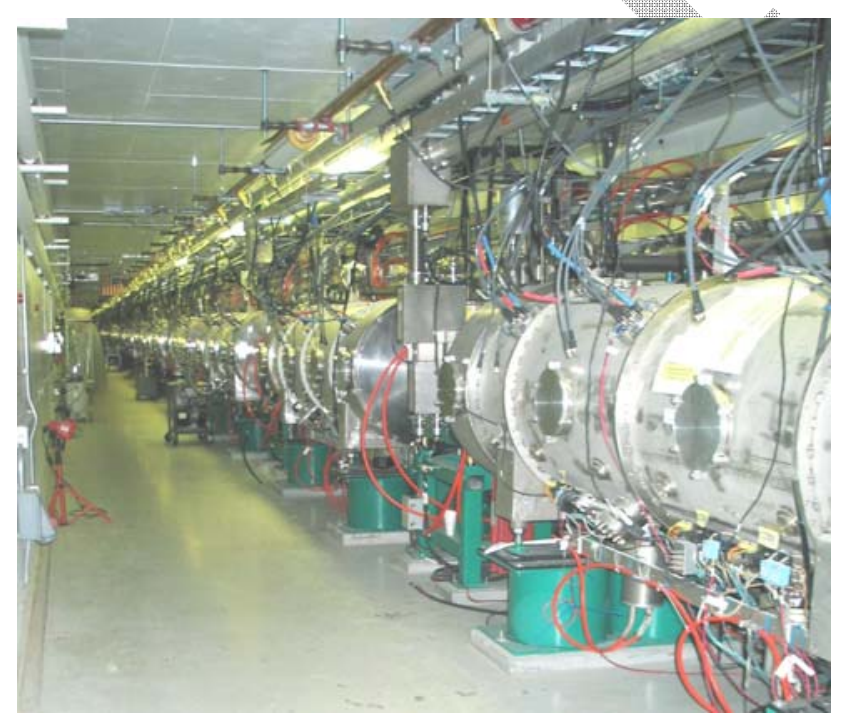

Figure 4. The Spallation Neutron Source (SNS) superconducting linac at ORNL.

SNS is the most recent large SRF based linac to be completed, Figure 4, and is specifically designed for $\mathrm{H}^{-}$ions (stripped to protons in the accumulator 
ring). It also has achieved $1 \mathrm{GeV}$ and $1 \mathrm{MW}$ of beam delivered to users but in pulsed mode with a $5 \%$ duty factor (6\% design). SNS has 81 independently powered $805 \mathrm{MHz}$ cavities in 23 cryomodules (11 medium beta, 12 high beta), with space reserved for additional modules to upgrade the energy to $1.3 \mathrm{GeV}$ and power to 2-3 MW. The design gradients were $10.1 \mathrm{MV} / \mathrm{m}$ for the beta 0.65 cavities and $15.8 \mathrm{MV} / \mathrm{m}$ for the beta 0.81 structures with $\mathrm{Q}_{0}$ of $5 \mathrm{E} 9$ at $2.1 \mathrm{~K}$. These gradients were achieved on average during cavity qualification and module testing during individual cavity commissioning, however high levels of dark current were observed that resulted in lower ensemble operating limits for the high beta cavities (see section 6). The SNS cavities were also operated at $4 \mathrm{~K}$ during initial commissioning at low duty factor with no apparent reduction in gradient performance [3].

Overall SNS has been a very successful project having been completed on time and on budget and having met all of the commissioning milestones to date, including delivering $1 \mathrm{MW}$ of beam on target during the 2009 run.

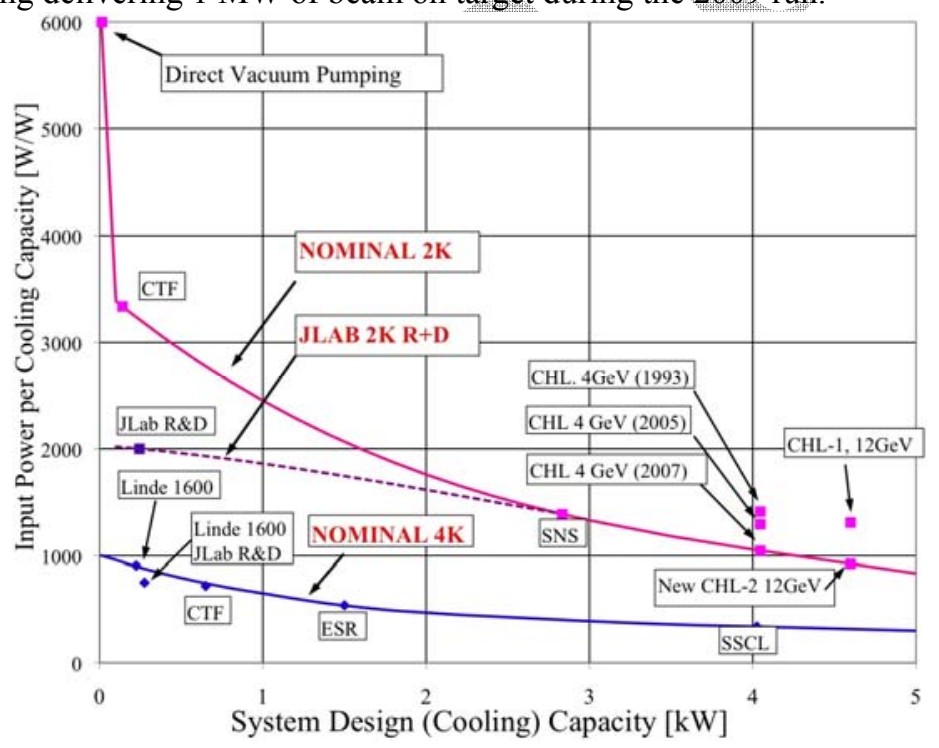

Figure 5. Typical efficiencies of existing and planned $2 \mathrm{~K}$ and $4 \mathrm{~K}$ cryogenic plants. 


\section{2K Cryogenic Plant Development}

Until recently most large $2 \mathrm{~K}$ cryogenic plants were designed for most efficient operation at their maximum capacity but quickly lost efficiency when turned down. Recent R\&D at JLab [4] has resulted in significant efficiency increases (e.g. 20-25\% improvement at $\sim 4.5 \mathrm{~kW}$ for JLab CHL-2), but also allowing good efficiency at high turn down ratio (as low as $34-40 \%$ of maximum output for the full Ganni cycle). This can result in up to a threefold power reduction when running such plant well below maximum capacity. The JLab floating pressure technology has been applied to a number of existing plants such as SNS, BNL and JLab CHL-1 with consistent improvements in efficiency resulting in very substantial savings in operating costs as well as reduced maintenance. Figure 5 shows some efficiency points for existing or planned machines along with nominal curves for $2 \mathrm{~K}$ and $4 \mathrm{~K}$ operation. Note that although the wall-plug power per cryogenic Watt is lower at $4 \mathrm{~K}$ than $2 \mathrm{~K}$ this is more than offset by increased SRF losses at the higher temperature for most presently produced SRF cavities, favoring $2 \mathrm{~K}$ (or lower) temperature operation for CW applications. If cavities could be made with much lower residual resistance than presently achieved then it might become favorable to operate low frequency RF cavities at $4 \mathrm{~K}$, greatly simplifying the plant and reducing capital costs. This is a subject of active investigation [e.g. 5].

\section{Cost Drivers and Technícal Risk}

Major cost drivers for any large SRF linac include the cryomodules themselves, RF power and controls, Cryogenic plant and distribution and tunnel and conventional facilities. Some typical parameters and estimated numbers from the JLab $12 \mathrm{GeV}$ upgrade project are shown in table 1 to give a feeling for the magnitude of these costs. As part of any overall project optimization these costs can be traded against each other to find the minimum capital cost, operating cost or combined capital plus some number of years operating. Two such recent studies are enlightening, the Cornell $100 \mathrm{~mA}$ ERL design study [6] and the UK new Light Source (NLS) conceptual design, a 1-pass CW linac [7]. Figure 6 shows the breakdown of these cost elements for the Cornell study. Of course these costs depend strongly on assumptions of cavity performance and gradient choice. Costs such as tunnel, cryomodule and controls (e.g. LLRF) typically vary linearly with tunnel length (i.e. inversely proportional to assumed gradient), while 
cavity RF power and cryogenic costs (which dominate operating costs as well as contributing significant fractions of capital expenditure), vary quadratically with gradient. Beam RF power cost is roughly constant for a given energy and current. The tradeoff of these types of cost tends to produce a rather broad cost minimum with respect to gradient, as shown for example in Figure 7 for the Cornell ERL.

Table 1. Some typical CW cryomodule parameters from the JLab upgrade conceptual design.

\begin{tabular}{|c|c|}
\hline Frequency & $1.5 \mathrm{GHz}$ \\
\hline Gradient & 15-20 MV/m CW ( $\sim 10 \mathrm{MV} / \mathrm{m}$ real-estate gradient $)$ \\
\hline $\mathrm{Q}_{\mathrm{o}}$ & $\sim 10^{10}$ at $20 \mathrm{MV} / \mathrm{m}$ has been demonstrated. $8 \times 10^{9}$ assumed. \\
\hline Module cost & $\sim \$ 2.6 \mathrm{M}^{*}$ \\
\hline $\begin{array}{l}\text { RF cost/module } \\
\text { Inc. LLRF }\end{array}$ & $\sim \$ 1.7 \mathrm{M}^{*} /$ cryomodule $(8 \mathrm{x} 13 \mathrm{~kW}$ RF stations) at $\sim 1 \mathrm{~mA}$ \\
\hline $\begin{array}{c}\text { Cryo plant } \\
4.5 \mathrm{~kW} \text { CHL-2 }\end{array}$ & $\sim \$ 30 \mathrm{M}^{*} / \mathrm{GeV}(\mathrm{CHL} 2)$ excluding distribution \& cold box \\
\hline Integrated cost/GeV@1 MW & $\sim \$ 7 \mathrm{M}^{*}$ (excluding tunnel \& conventional facilities) \\
\hline
\end{tabular}

*FY08 Estimate, loaded dollars, no contingency. Actual $12 \mathrm{GeV}$ project costs will be known soon.

Technical risk also plays a significant factor in gradient choice. Given the relatively broad cost optimum there is little or no penalty in choosing a gradient at the low end of the curve, e.g. closer to $15 \mathrm{MV} / \mathrm{m}$ than $25 \mathrm{MV} / \mathrm{m}$. This will diminish the risk of degraded machine performance from field emission, reduce coupler power requirements and (in all likelihood) provide operating overhead in case of installed cavities being out of service. However more cavities will mean more systems to maintain, slightly stronger HOM damping requirements, proportionally more emittance growth from wakefields and coupler kicks (if present), etc. All these factors should be taken into consideration in the overall optimization. However it is encouraging that these gradient levels have already 
been demonstrated in large numbers of cavities in operating facilities. It should be noted that this optimization for CW operation is fundamentally different than for a pulsed energy-frontier machine like ILC, where the highest practically realizable gradient is the cost optimum. The case of the CEBAF upgrade cryomodules is also slightly different in that the tunnel already exists and there are only ten vacant zones available for new cryomodules. This forces the spec to be closer to $20 \mathrm{MV} / \mathrm{m}$, however this performance has been demonstrated and is still conservative compared to machines like ILC and XFEL.

Other technical risks include beam break-up and other instabilities. Although these are rather well understood and modeled there remains some disagreement over the need for HOM damping in high energy but modest current machines. A more significant risk arises from beam loss in the linac that can cause a serious problem of activation. This has already been observed at SNS and although they have been able to operate below the $1 \mathrm{~W} / \mathrm{m}$ nominal loss for hands-on maintenance they are doing so at considerably lower average current than needed for a very high power CW proton driver. Extensive beam dynamics modeling has not yet identified or reproduced the source of the losses although speculation is that at least some fraction is coming from longitudinal losses out of the RF bucket originating from mismatches upstream in the machine.

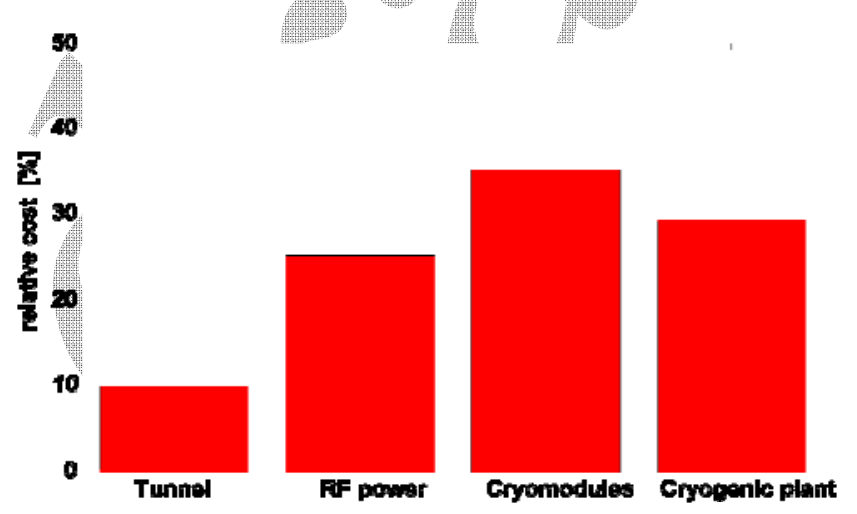

Figure 6. Cost distribution of major systems in the Cornell $100 \mathrm{~mA}$ ERL design study. 


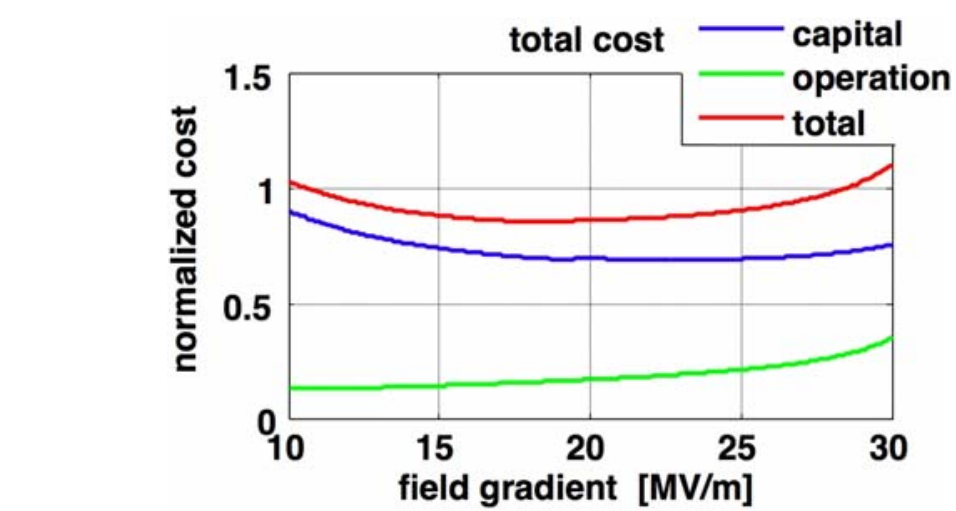

Figure 7. Typical cost variation with gradient for a CW linac (Cornell $100 \mathrm{~mA}$ ERL study).

\section{Other Parameter Choices}

\subsection{Current}

Depending on the application there may be a possible trade-off between current and energy for a given beam power on target, however most new $\mathrm{CW}$ projects under consideration seem to be seeking at least $1 \mathrm{GeV}$ and 1 $\mathrm{mA}$, with up to $8 \mathrm{GeV}$ and $30 \mathrm{~mA}$ being discussed [8], Figure 8. Cavities exist that are designed with sufficient HOM damping for $100 \mathrm{~mA}$ or above so this should not be a limitation. Coupler power becomes a factor at high current but this is a matter for careful R\&D rather than a hard limit.

\subsection{Frequency}

Exact frequency choices for operating machines are often a result of history such as existence of proven cavity designs, RF sources (e.g. the UHF TV band is very popular), or synchronism with other accelerators. In general BCS theory [7] would predict more efficient operation at lower frequencies with the added benefit of (typically) larger beam apertures and, possibly, fewer cavities for a given active length. However given the typical residual resistances of presently produced SRF cavities the actual benefit in efficiency is somewhat reduced (although the other benefits remain). The downside, at least for elliptical cavities, is the increased size and cost. Handling considerations are also a factor for multi-cell cavities, setting a practical lower limit in the $500-700 \mathrm{MHz}$ range. Spoke cavities and other compact structures are becoming increasingly popular, especially for the 
low beta end of the linac where they are also more efficient than squashed ellipticals. Beta $=1$ spoke cavities are also under development and typically are about half the diameter of an equivalent elliptical cavity at the same frequency, however they also have higher peak surface fields for a given accelerating gradient. Many projects seem to be converging on a combination of quarter-wave or spoke cavities at around $350 \mathrm{MHz}$ for the low beta part of the linac and one or more flavors of elliptical cavities at around $700 \mathrm{MHz}$ for the high-energy end. The transition is typically at about beta $=0.5$ or higher. The number of cells or interaction gaps per cavity and the number of different beta values are subjects for detailed beam dynamics optimization.

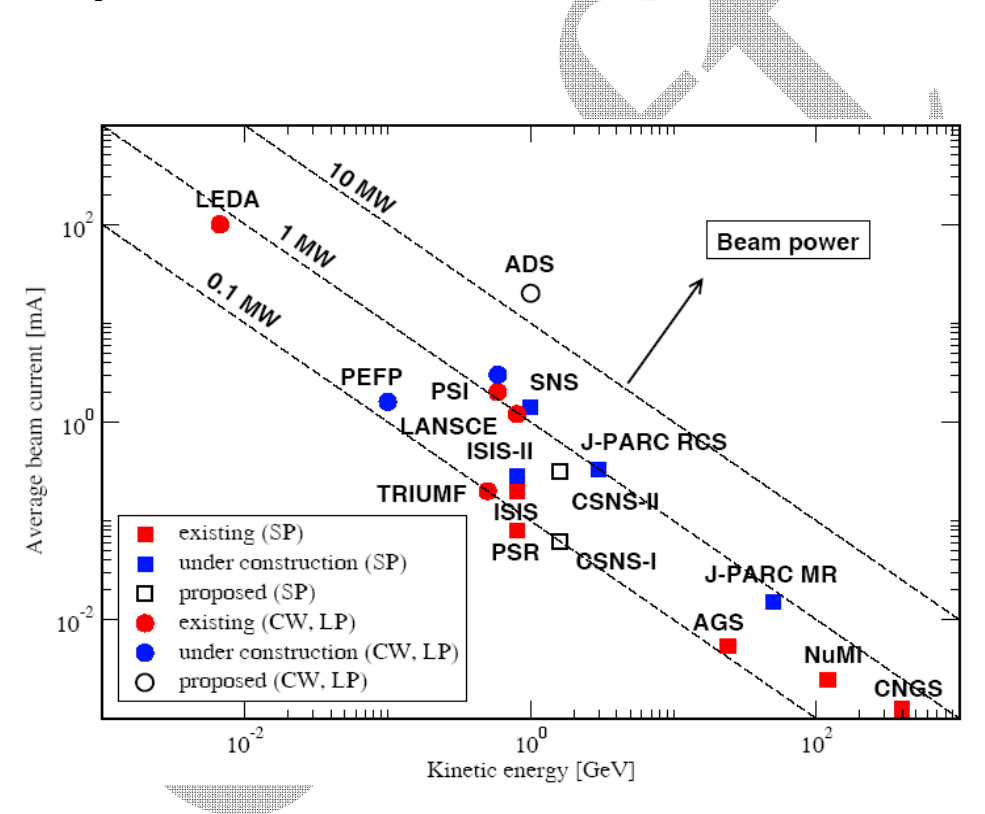

Figure 8. Landscape of existing and planned high power proton sources.

\subsection{Operating Temperature}

For high-gradient CW operation cavities are normally operated in superfluid helium $(<2.18 \mathrm{~K})$ to benefit from the increased heat transport capability, however both the maximum superfluid heat transport limit and the RF losses are strong functions of temperature in this range. Depending 
on the achieved residual resistance of the cavities temperatures as low as $1.8 \mathrm{~K}$ or even $1.6 \mathrm{~K}$ may be attractive. Figure 9 shows an example of how the BCS surface resistance asymptotes to the residual resistance (assumed to be $10 \mathrm{n} \Omega$ ) as the temperature falls below about $2 \mathrm{~K}$ [5]. Clearly in this case there is limited benefit from going much further down in temperature. Any optimization needs to take into account the specific cryogenic system operating characteristics and environment, e.g. long distribution lines and high mass flow may limit the practically attainable minimum pressure in the helium bath. CEBAF was designed for and operates at $2.07 \mathrm{~K}$, however the actual dependence of operating efficiency (and therefore cost) on temperature has never been mapped. This would be a worthwhile experiment providing it could be performed without affecting user operations.

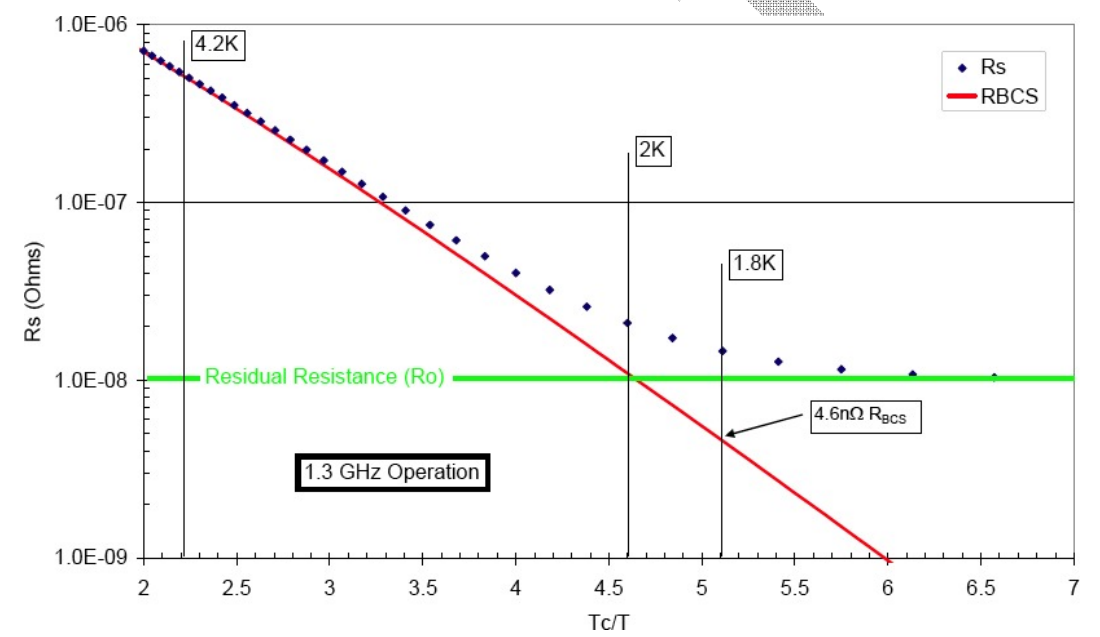

Figure 9. Example of total surface resistance vs. temperate below $\mathrm{T}_{\mathrm{c}}$. Note deviation from BCS resistance to residual resistance at low temperature $\left(\mathrm{T}<<\mathrm{T}_{\mathrm{c}}\right)$.

\section{SNS Experience}

As mentioned previously the SNS is the most recent example of a large SRF linac and the first designed as a MW class proton source. The decision to use SRF was taken late in the project timeline so there was only a short time available to design and prototype the cavities and cryomodules before 
launching series production. In light of this the project has been remarkably successful and has met all of its performance milestones to date. However several issues arose during the production and commissioning that are worth taking note of.

\subsection{Initial Cavity Qualification}

Initial cavity qualification was quite problematic with persistent field emission and/or multipacting in many of the cavities. On average it took between two and three processing cycles to get the cavities to qualify in vertical test. Suspicion fell on the effectiveness of the high-pressure rinse system and water quality although investigation at the time and since seems to indicate that probably the water purity was not the issue. The effectiveness of the HPR spray jet at the equator of the $805 \mathrm{MHz}$ cavities has since been measured and found to be significantly less than that for smaller cavities, however it may not be the only factor. Due to the tight production schedule it was difficult to resolve these issues in real time and although many changes were made to the process during the run, such as extended rinse times and different assembly procedures, the net cavity yield was not substantially higher at the end of the project than at the beginning. Another feature of these cavities with BCP process was a strong high-field Q slope even in the absence of field emission. Figures 10 and 11 show some typical curves from medium beta and high beta cavities [10]. This made it harder to meet the $\mathrm{Q}$ specification even if the gradient limit was acceptable, especially for the high beta cavities. Many of the qualified cavities still had high levels of field emission at the operating gradient even though they met both the gradient and $\mathrm{Q}_{0}$ specifications.

Figure 12 shows the residual resistance of the high beta cavities estimated from the measured low-field $Q_{0}$ values [11]. The average value is about $8 \mathrm{n} \Omega$ but there is a wide spread in values. 


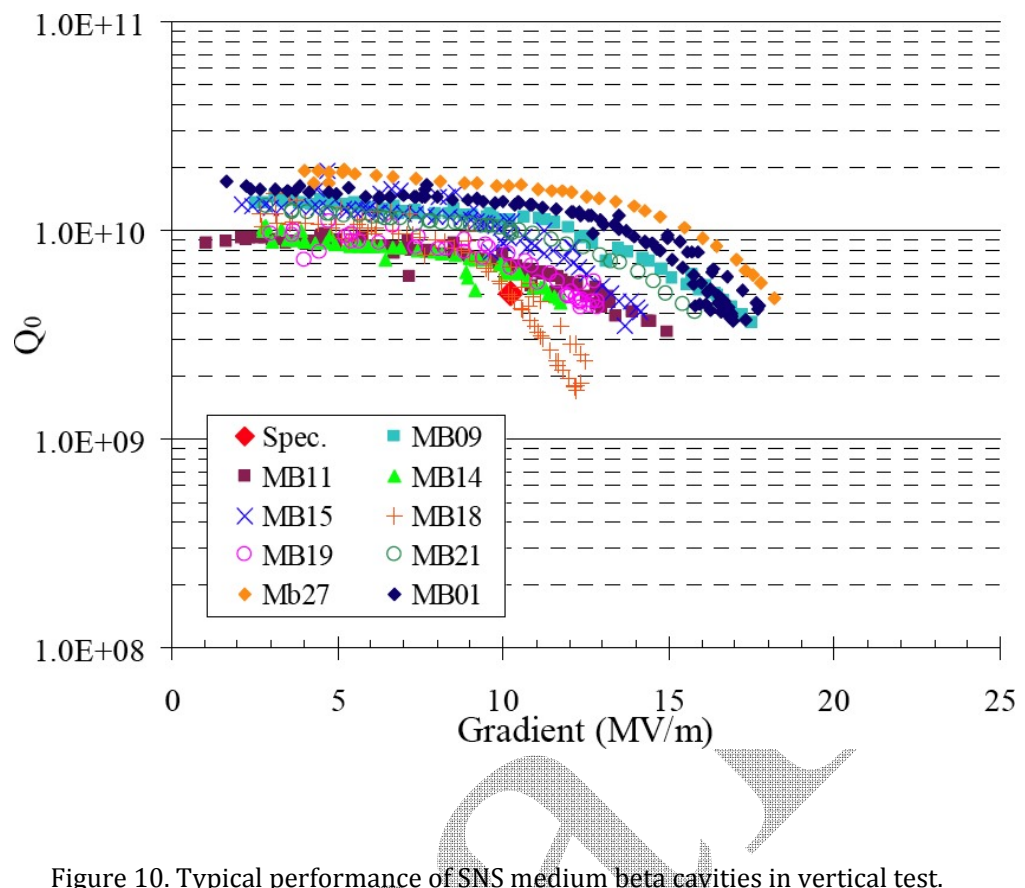

Figure 10. Typical performance of SNS medium beta cavities in vertical test.

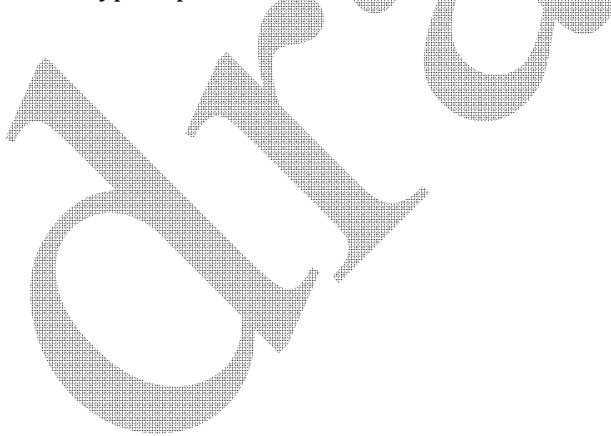




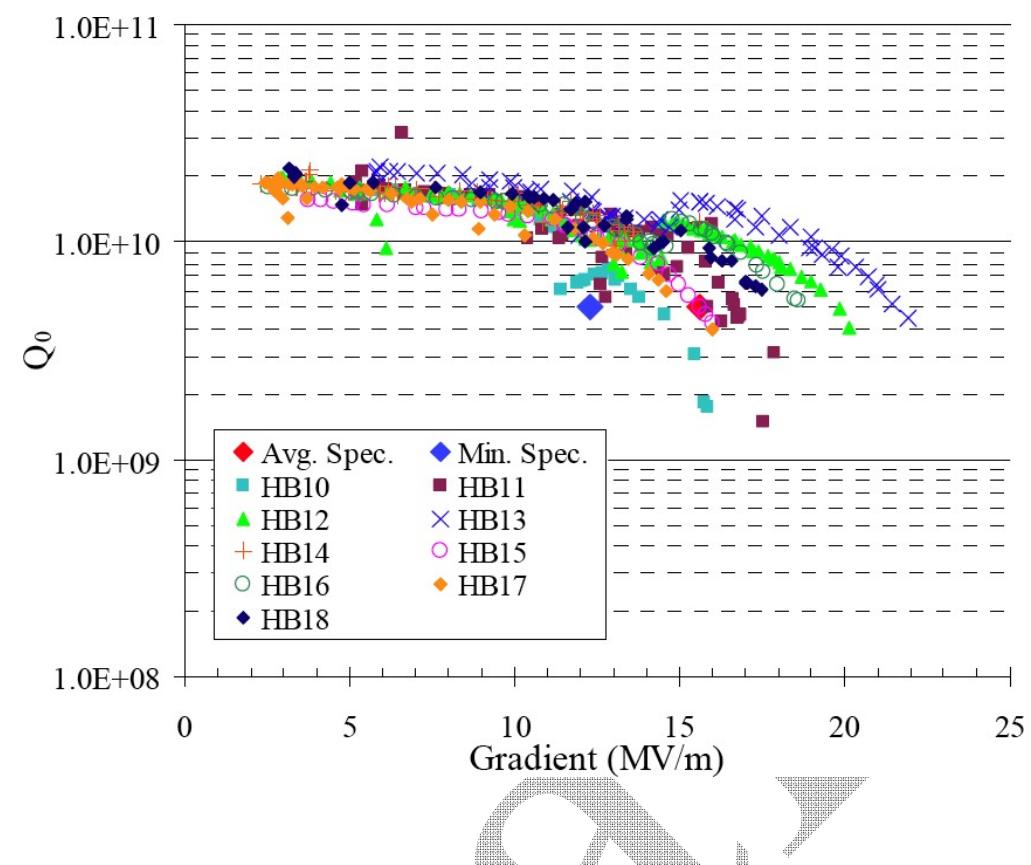

Figure 11. Typical performance of SNS high beta cavities in vertical test.

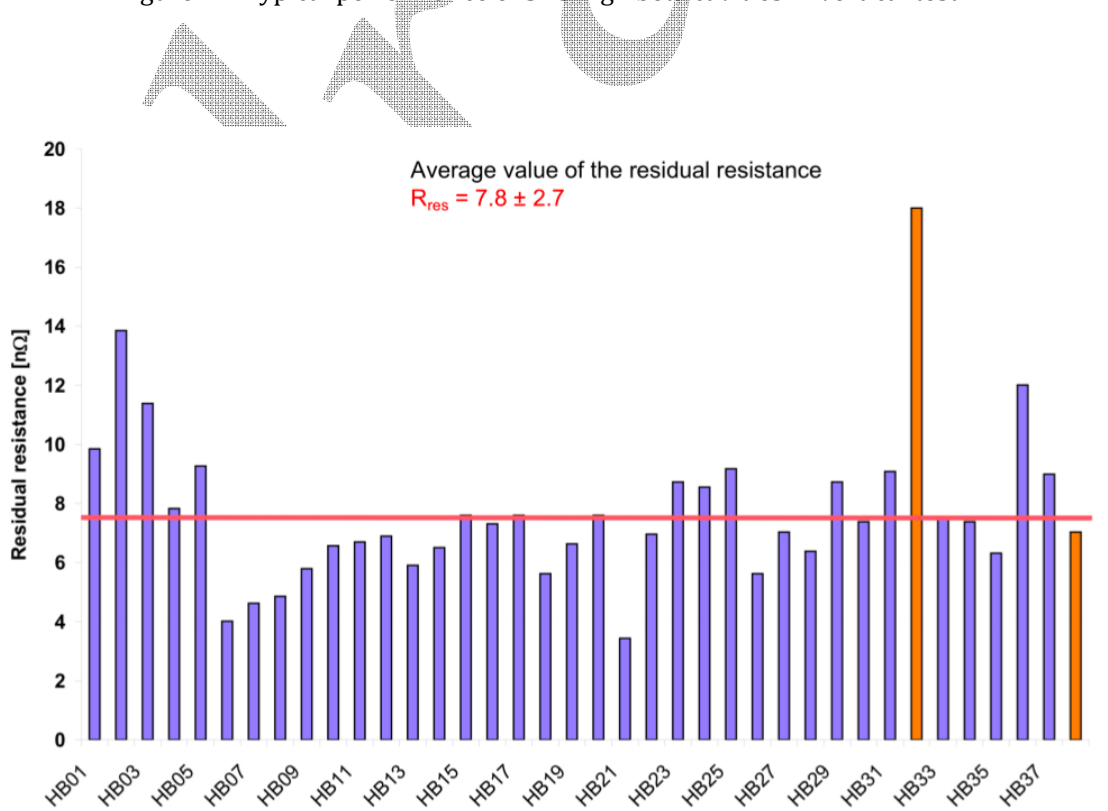


Figure 12. Residual resistance of the SNS high beta cavities estimated from the low-field Qo.

\subsection{Cavity Performance in Modules:}

Early production cryomodules were tested at JLab before shipping to Oak Ridge, affording an opportunity to compare VTA performance with horizontal performance in the cryomodule, Figure 13 [10]. Since the cavities underwent an additional HPR and assembly for the string some scatter in the data is not unexpected, however the average performance in the cryomodule was not degraded compared to VTA, in fact it was slightly better. Thus the cryomodules were delivered to SNS with high expectations of successful operation

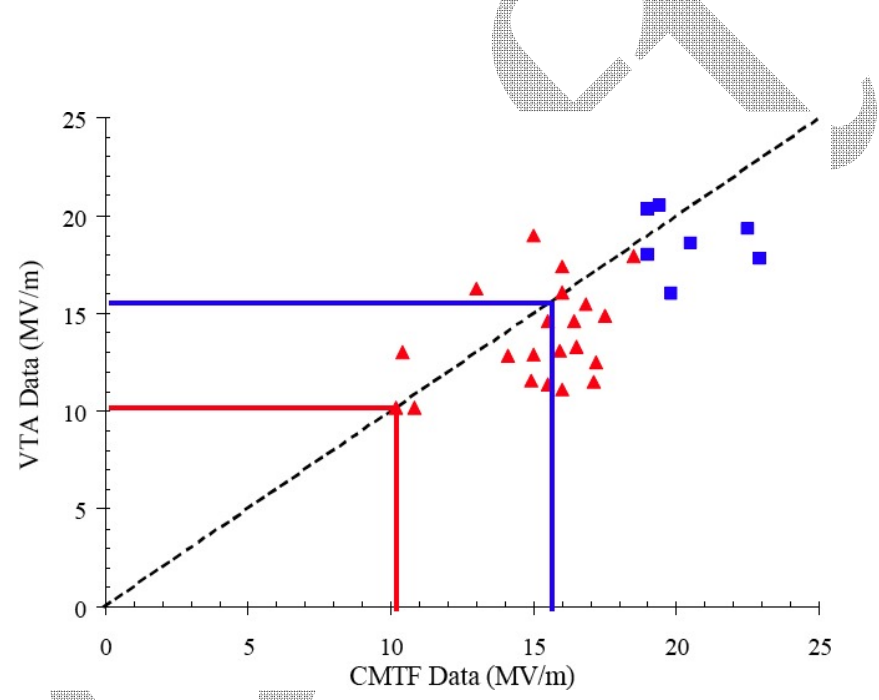

Figure 13. SNS cavity data in vertical test vs cryomodule. Gradient at $\mathrm{Q}_{0}=5 \times 10^{9}$, as measured in the VTA and CMTF, for the medium- $\beta$ (triangles) and high- $\beta$ (squares) cavities.

\subsection{Cavity Performance in Initial Ensemble Operation:}

Initial commissioning of the modules as an ensemble at Oak Ridge uncovered a number of issues with HOM coupler tuning, power coupler vacuum and some end group thermal limitations from dark current interception that had not been apparent in single cavity limits. As planned many of the cryomodules were not tested at JLab and received their first commissioning at Oak Ridge. As a necessary first step many of the cavity gradients were simply turned down in order to get the linac to run stably. 
This unfortunately led some people to speculate that there might be a systemic problem in the linac that would prevent SNS from achieving its design energy. Fortunately calmer heads prevailed and after much careful diagnosis and tuning most of the lost gradient has been recovered and the machine is functioning well for users [12]. A few cavities with hardware problems were repaired or removed. The cavities in low beta section of the linac are now operating above the design specification on average. In the high beta section the individual cavity limits now also exceed the specification on average but the cross talk from captured dark current means the ensemble average is still slightly below spec. This is an effect that it was not possible to observe during the prototyping and module testing phases. The net result is that SNS is operating very well and the SRF linac is a very minor contributor to down time. However the operational margin is less than desired. There is an active plan under way to rework some more cavities to provide spare capacity.

\subsection{Prospects for Improvement}

The standard process for SNS cavity qualification employed buffered chemical polishing (BCP) etching for both the bulk material removal as well as final cavity processing. It has been observed that repeated BCP processing can cause the high-field $Q$ slope to become more severe, correlated with visible roughening of the surface. A few cavities were electopolished early in the project with encouraging results but VTA tests at that time were still often dominated by field emission so potential improvements were somewhat masked. Due to schedule and budget pressures the project decided not to continue EP development for the remainder of the production cavities.

Recent success with EP of ILC cavities in the same JLab facilities suggests that in the absence of field emission there is a significant benefit from EP both in gradient reach as well as elimination of the $\mathrm{Q}$ slope (after $120^{\circ} \mathrm{C}$ baking) [13]. This has revived interest in EP as a possible process for the SNS power upgrade project, however the question mark over effectiveness of HPR in these cavities remains.

Very recently JLab has experimented with final light EP on several 7-cell 1.5 GHz cavities that have had bulk BCP, some with repeated cycles of final BCP [14]. As can be seen in Figure 14 the results are very encouraging with only 30 um removal and $120^{\circ} \mathrm{C}$ bake for 48 hours the performance improved 
dramatically and the high field Q slope was virtually eliminated. This further supports the theory that the surface roughness on small length scales is a contributing factor to the Q slope in BCP'd cavities. The residual resistance also appears to be quite low suggesting advantage may be gained by lowering the operating temperature. There is some interest at ORNL in testing this process on spare SNS cavities.

Improvements to the cavity geometry and construction may also be worthwhile. It is known that there is a persistent multipacting barrier in the high beta cell shape and this could be reduced significantly by altering the cell profile at the equator region. Also end group cooling could be improved or higher RRR material could be used to help mitigate the thermal issues from dark current heating. Concerns about possible multipacting in the HOM couplers could be addressed by design changes or possibly these could be eliminated altogether.

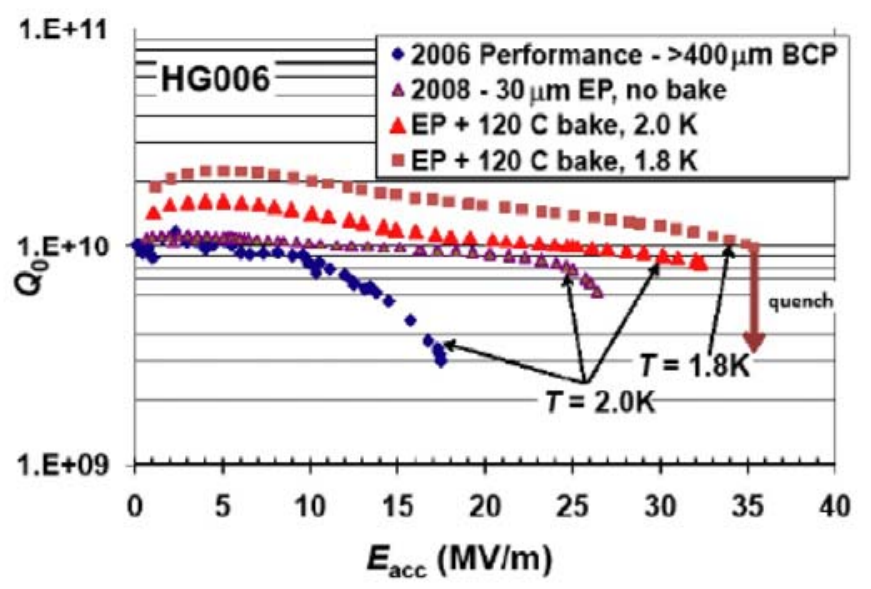

Figure 14. Performance of JLab 7 -cell cavity HG006 after nominal BCP (purple triangles), after subsequent heavy BCP (blue diamonds), and after only $30 \mu \mathrm{m} \mathrm{EP}+120^{\circ} \mathrm{C}$ bake (red triangles). Test at $1.8 \mathrm{~K}$ (brown squares) suggests cavity has low residual resistance.

\section{Candidate Structures for new High-Power CW Machines}

A number of candidate cavity types either exist or are in development in addition to those already proven in existing machines. Half-wave and spoke resonators in a variety of styles are rapidly becoming the default choice for the low beta sections of new machines. For the higher energy sections elliptical cavities are still popular, although high beta and beta=1 spoke cavities are under development. At beta above about 0.5 elliptical cavities 
tend to have good efficiency and lower peak surface fields than spoke types. At lower betas spoke resonators typically have the advantage. Elliptical cavities tend to have larger apertures, which might be an advantage for minimizing halo interception, but spoke resonators are significantly more compact for a given frequency and tend to have better microphonic performance and higher filling factor in the cryomodule.

\subsection{Low Beta}

An example of a low beta single spoke resonator is the "SSR1" [15], Figure 15 developed for the HINS project. A prototype of this cavity has been built and tested with excellent results.

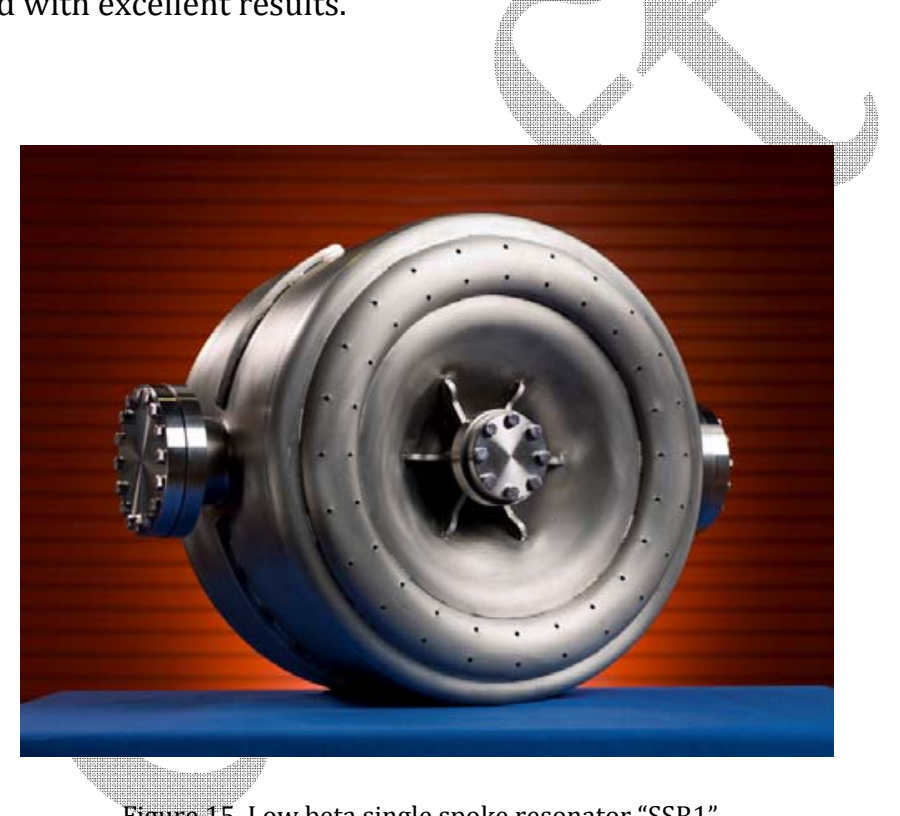

Figure 15. Low beta single spoke resonator "SSR1".

\subsection{Medium Beta}

Examples of medium beta multi-spoke cavities include the $345 \mathrm{MHz}$ beta 0.5 and beta 0.62 triple spoke resonators developed at ANL [16], Figures 16,17 . Originally designed for $4 \mathrm{~K}$ operation the performance of these cavities at lower temperature is so good that it would clearly be more efficient to operate these cavities CW at $2 \mathrm{~K}$ or even lower, see Figure 18. 
Although elliptical designs have been prototyped at beta 0.5 or below, triple spoke resonators seem to be the popular choice. At around beta $=0.6$ multi-cell elliptical cavities are a viable option (e.g. SNS low beta $=0.61$ ).

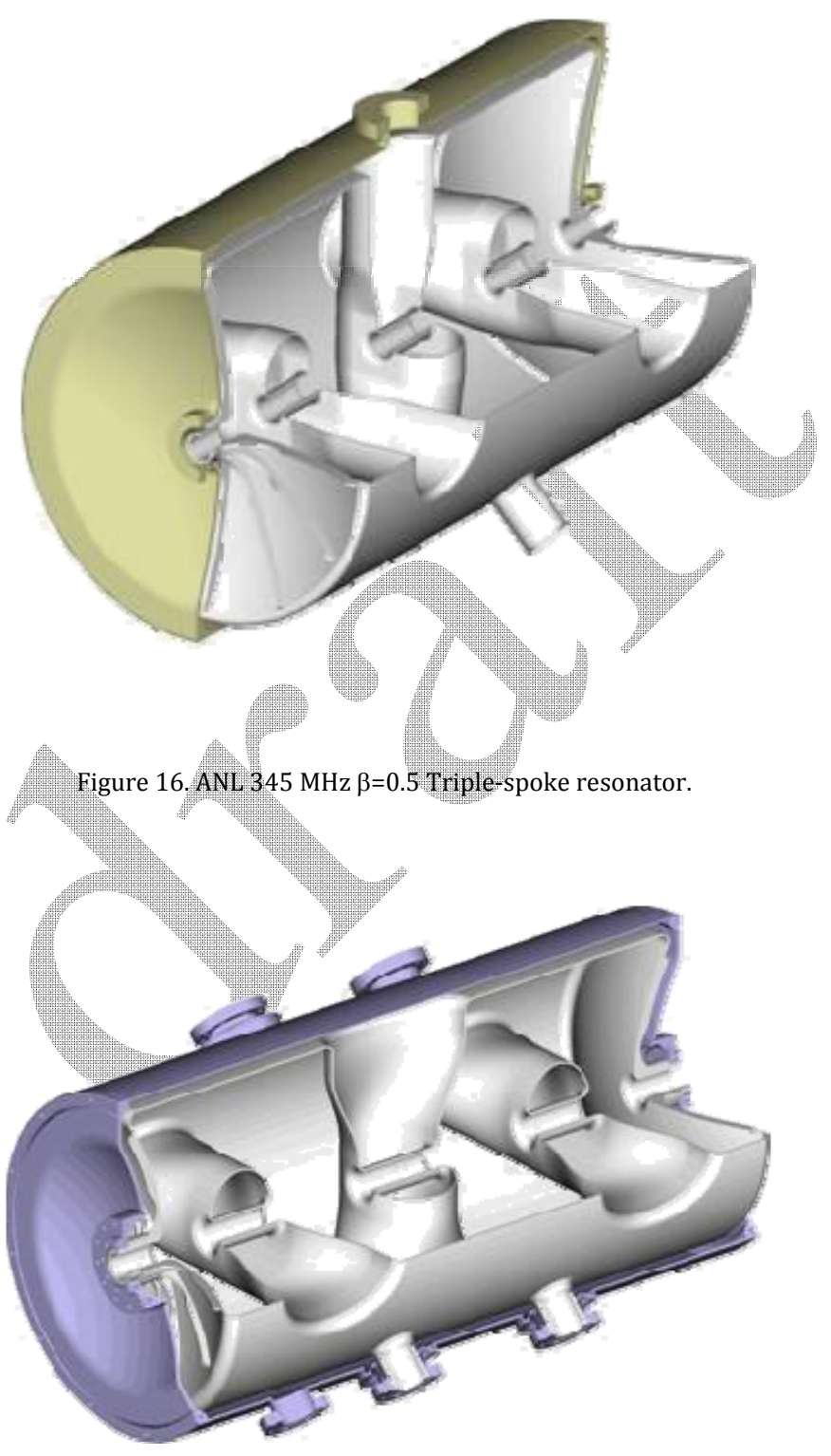


Figure 17. ANL $345 \mathrm{MHz} \beta=0.62$ Triple-spoke resonator.

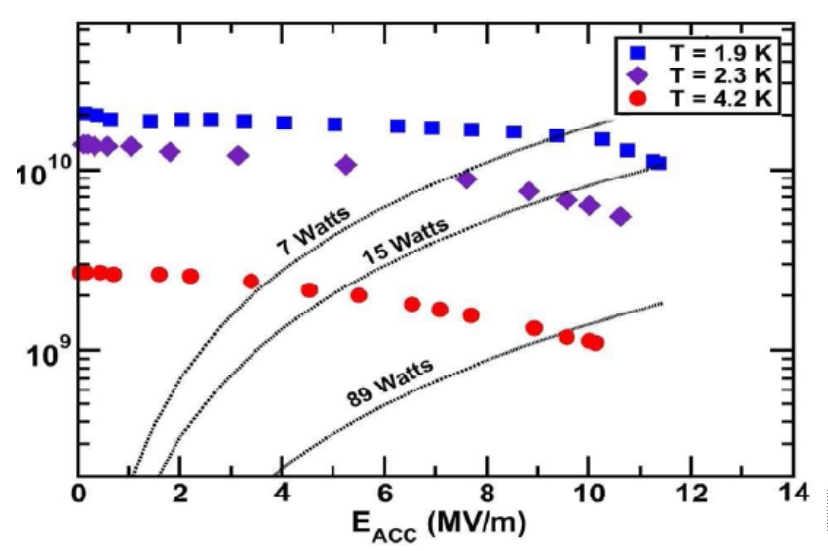

Figure 18. Cryogenic test performance of ANL medium beta triple spoke resonator at various temperatures.

\subsection{High Beta}

At beta approaching unity elliptical cavities are presently the only proven choice, although high beta spoke resonators are being actively developed. The SNS beta $=0.85$ cavities have demonstrated performance in the right range for a high current CW proton machine, with potential for further improvement. Prototypes such as the $704 \mathrm{MHz}$ BNL electron cooling cavity [17] and $750 \mathrm{MHz}$ JLab high-current cavity [18], although designed for very high current electron beams, could also be candidates. They have the advantage of very large beam apertures and very strong HOM damping (although this is probably overkill for even the highest power proton driver application). Figure 19 shows how a beta $<1$ version of the JLab cavity might look and Figure 20 shows a family of such cavities that could share a common end group design. The BNL ECX cavity was qualified at JLab to 20 $\mathrm{MV} / \mathrm{m}$ with $\mathrm{Q}_{\mathrm{o}} \geq 1 \mathrm{E} 10$ while a single cell beta $=1$ version of the JLab highcurrent cavity shape also showed excellent performance on the first test, Figure 21.

Based on the SNS experience some effort was put into lowering the impact energy in the multipacting zone of the new JLab cavity shape, see Figure 22. No 
sign of multipacting was observed in the VTA test of the high-current single cell. VTA testing of the 5-cell version is planned in the near future.

Any of these concepts can be scaled to a chosen design frequency although staying in the UHF TV band has advantages in terms of available high power RF sources and proven high-power coupler and RF window designs [19, 20].
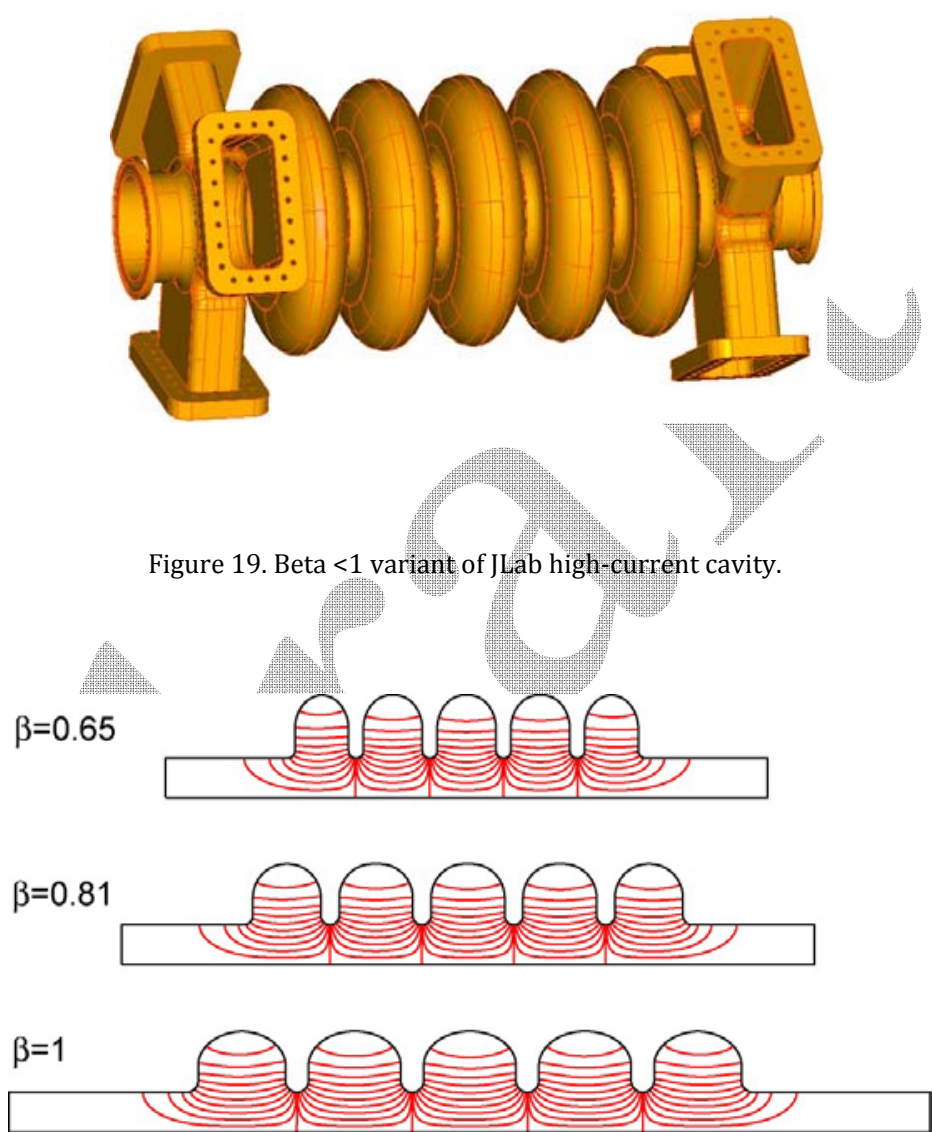

Figure 20. Family of cavities based on the JLab high-current cell shape that could use a common end group and coupler. 


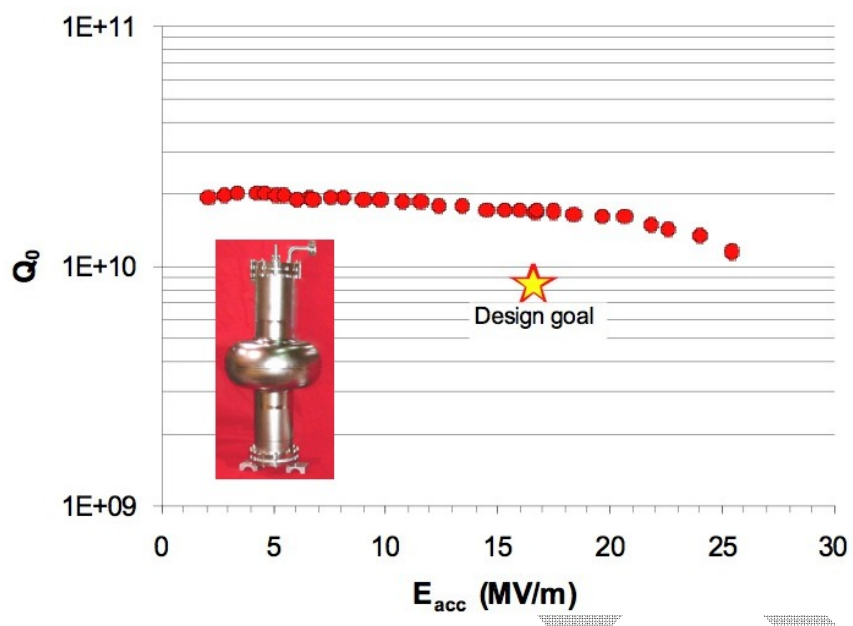

Figure 21. First vertical test result of JLab $750 \mathrm{MHz}$ hïgh-current single cell cavity.

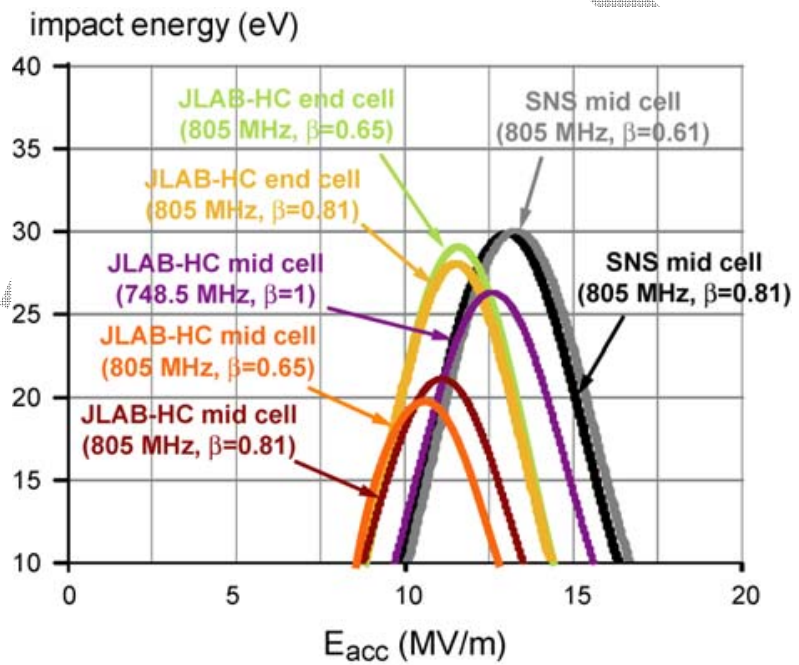

Figure 22. Impact energy of multipacting electrons as a function of gradient in various cell shapes. Flattening the equator region of the cell significantly reduces the impact energy and therefore the likelihood of secondary emission.

\section{Cryostat Options}

While a lot of attention has been focused on the well-developed TESLA type cryomodule it should not be forgotten that there are other proven designs 
in existence, many of which are already optimized for CW operation. Examples include SNS, ANL (ATLAS and HINS types), KEK, SACLAY, CERN (LEP and LHC), and CEBAF (original and upgrade types), and more are in development. Depending on the chosen scheme for cryogenic segmentation (e.g CEBAF and SNS are fully segmented with external cryogenic distribution, ILC is minimally segmented with internal distribution), a large internal gas return pipe may not be required or even desirable. The TESLA type module uses the gas return pipe as the cold mass support. Other schemes use a separate support structure or even the vacuum vessel itself for support and alignment of the cold mass, allowing simplified internal helium supply and gas return but requiring external bulk cryogenic distribution. The fully segmented option has great advantages in terms of operability and maintainability of the cryomodule inventory. The primary motivation for internal distribution in the TESLA type module is to minimize static heat load, which is much less of a concern in the CW case where dynamic losses dominate.

Figure 23 shows a concept for a cryomodule containing low beta spoke resonators [20] while Figure 24 shows a concept for the JLab high-current cryomodule based on the CEBAF upgrade/SNS configuration [21]. Figure 25 shows a version of the XFEL type cryomodule. The changes necessary to operate this type of module $\mathrm{CW}$ are well understood and have largely been addressed by various projects aimed at $\mathrm{CW}$ light source or ERL applications [22]. Should any part of a CW proton linac be chosen to operate at above $1 \mathrm{GHz}$ this could be a viable option.

It is possible to conceive of a common modular cryostat sized to support single and triple spoke resonators e.g. at $352 \mathrm{MHz}$ and one or more families of elliptical resonators at twice that frequency, e.g. $704 \mathrm{MHz}$ using the same support structure and cryogenic distribution throughout and a high degree of commonality in ancillary components such as tuners and couplers. This could provide significant economies of scale and ease of maintenance compared to multiple dissimilar families of cryomodules. 

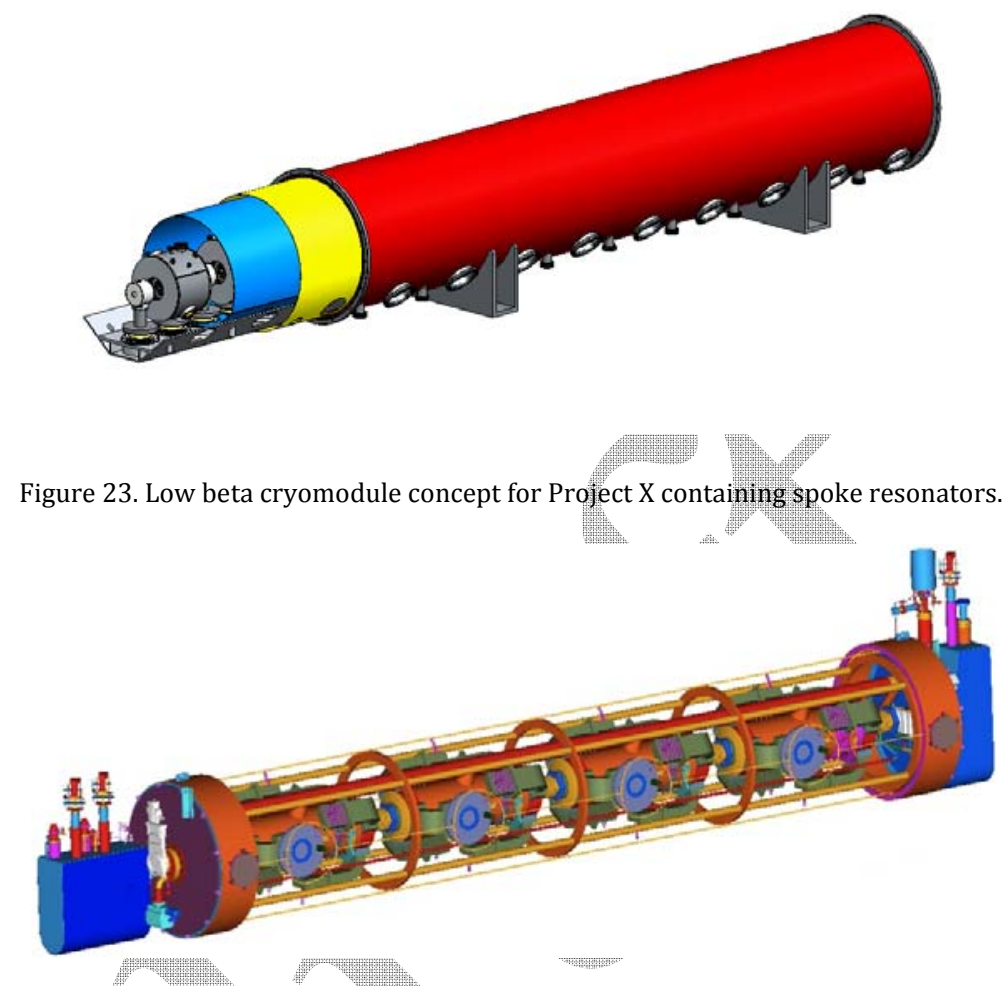

Figure 24, JLab 750 MHz ERL module (based on modified SNS layout).

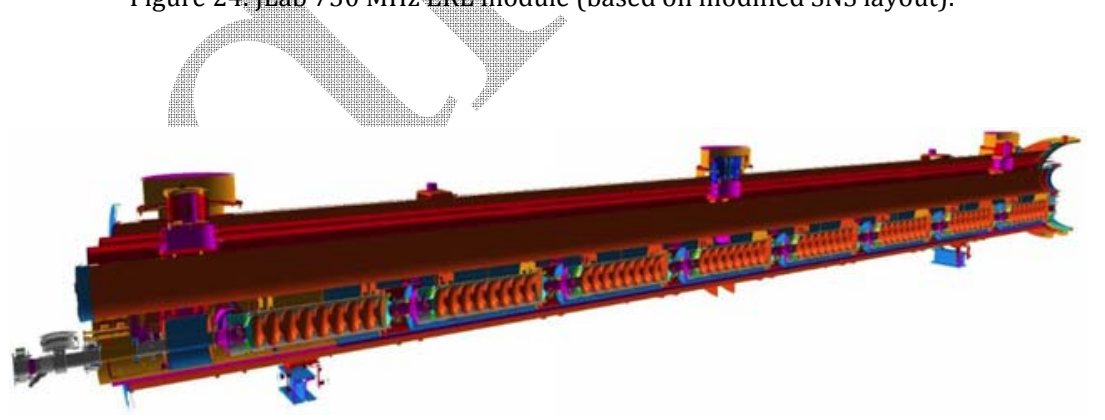

Figure 25. XFEL-type cryomodule modified for CW with larger cryogenic piping (chimney, 2phase line), higher-power FPC and modified temperature stabilized HOM probes. 


\section{Topics for further R\&D}

A significant cost driver common to all of the CW designs discussed above is the residual RF loss in the superconductor. Understanding the origins of the residual resistance and developing robust protocols to achieve more efficient CW operation would pay large dividends in both capital and operating costs. The wide scatter in observed residual resistance in presently produced cavities indicates that some property of the resultant surface is not being well controlled. This is a complex issue of materials, chemistry and morphology but is fertile ground for significant advancement.

Elimination of field emission is a prerequisite to achieving such benefits but based on SNS experience it remains a challenge in large low frequency cavities.

Microphonics overhead may be a concern for lightly beam loaded cavities but becomes less of a factor as average beam power increases and optimal Qext is reduced. However understanding the sources of excitation and system response may yield improvements in stability and efficiency. Tuning of cavity and support structure stiffness, intelligent design of tuners and application of active feedback may all be viable avenues to address these issues.

A major concern and high priority for further work should be to understand the origins of beam halo and loss mechanisms. Activation of components from beam loss may be a significant performance-limiting phenomenon until this is understood and mitigated.

A demonstration of the technology on a significant scale with beam (e.g. a CW version of HINS) is highly desirable, especially in light of the SNS experience where demonstrated individual component performance was not sufficient to guarantee reliable system operation.

BBU analysis and appropriate HOM damping (including real couplers and loads) should be performed in coordination with beam dynamics requirements. A comfortable safety margin in BBU threshold is advisable but it is possible to be too conservative at the cost of overall system efficiency.

Low trip rate and rapid beam recovery (faster than target thermal time constants) will be very important for some applications such as ADS. Standard reliability engineering techniques such as built-in redundancy and MTBF analysis may go a long way in this regard however safe automated fast beam recovery without operator intervention may take some effort to develop. Experiments at CEBAF have indicated that for many fault conditions this is 
technically feasible but so far it has not been implemented (JLab policy requires that beam restoration always requires an operator action).

\section{Conclusions}

Facilities such as CEBAF and SNS operating with MW-class beam power demonstrate that CW SRF is a viable technology for very high power proton drivers. Suitable prototype cavities exist or are in development at multiple institutions for all necessary beta ranges. No "show stoppers" to operating in CW mode at high beam power have been identified. Robust high-power couplers must be used and high overall reliability must be engineered into the machine design. The main challenges may be beam halo, beam loss in the linac, minimizing trip rate and reducing recovery time to well below the thermal time constant of target system components. Cavity operating $Q_{0}$ (limited by the SRF residual resistance) has a significant leverage on cost. The origins of this residual loss are presently poorly understood but under active investigation. The "cost optimal" design configuration (gradient, frequency, temperature, structure type, etc) depends strongly on assumptions and a full multi-variable optimization using best available world scaling data would be worthwhile.

\section{Acknowledgments}

The author would like to thank D. Arenius, G. Ciovati, J. Delayen, S. Henderson, P. Kneisel, M. Liepe, J. Mammosser, F. Marhauser, P. McIntosh, T. Nicol, P. Ostroumov, J. Preble and C. Reece, J. Wei for useful advice and discussion on this subject and for kind permission to reproduce certain of the included Figures. The author would also like to thank the organizers of the AHIPA workshop for providing a unique and stimulating forum to present and discuss these ideas. This paper is based on a presentation of personal opinions by the author. These opinions are not necessarily shared by Jefferson Science Associates or the DOE, but they should be.

\section{References}

1. "The Continuous Electron Beam Accelerator Facility", H. A. Grunder, J. J. Bisognano, W. I. Diamond, B. K. Hartline C. W. Leemann, J. Mougey, R. M. Sundelin, R. C. York, proceedings of PAC 87. 
2. "Performance of the LEP2 SRF System", D. Boussard, proceedings of PAC97

3. "Thermal Stabilities and Optimal Operating Parameters for the Oak Ridge Spallation Neutron Source Superconducting Linear Accelerator", Sang-ho Kim, Isidoro Campisi, PRST-AB 10, 032001 (2007).

4. Dana Arenius, private communication (presented at the 2008 JLab internal Science and Technology review).

5. "High Field Q-slope and the Baking Effect", Gianluigi Giovati, Proc 14th International Conference on RF Superconductivity (SRF2009) Sept. 20 $-25,2009$, Berlin, Germany.

6. "SRF System Optimization Process", M. Liepe, Proc. ERL09, Cornell University, Ithaca, NY, USA, June 8-12, 2009.

7. "New Light Source (NLS) Project: Seience Case And Outline Facility Design", J. Marangos, et. al, 7/09, http://www.newlightsource.org.

8. "China Spallation Neutron Source Design", I. Wei et. al., Proc. APAC 2007, Raja Ramanna Centre for Advanced Technology (RRCAT), Indore, India]

9. "Theory of Superconductivity", John Bardeen, L.N. Cooper, J.R. Schrieffer, (Illinois U., Urbana) . Jul 1957. Published in Phys.Rev.108:1175-1204,1957.

10. "Performance Overview of The Production Superconducting RF Cavities for the Spallation Neutron Source Linac", J. Delayen, J. Mammosser, and J. P. Ozelis, Proc. PAC05, Knoxville, TN.

11. Gigi Ciovati, private communication to be published?

12. "The Spallation Neutron Source Linac: Performance and Operational Experience", Stuart Henderson, presentation at the 2009 Linear Collider Workshop of the Americas, LCWA 2009, September 29, 2009. http://panda.unm.edu/LCWA09 
13. "Overview of High Gradient SRF R\&D for ILC Cavities at Jefferson Lab", Rongli Geng, JLAB-ACC-09-1086, Proc. 14th International Conference on RF Superconductivity (SRF2009), Sept. 20 - 25, 2009, Berlin, Germany.

14. "Improving Gradient And Q Performance of BCP Etched Multi-Cell Cavities by Applying a Light EP", R.L. Geng et. al., Proceedings of SRF2009, Berlin, Germany, 2009.

15. "325 MHz Cryomodule Design Status and Plans", Thomas H. Nicol, presentation at the Project X collaboration meeting, FNAL, September 11-12, 2009.

16. "Triple-Spoke Cavities for a CW Proton Linac", P.N. Ostroumov, presentation at the Project X collaboration meeting, FNAL, September 11-12, 2009.

17. "Some Aspects Of $704 \mathrm{MHz}$ Superconducting RF Cavities", R. Calaga, Proceedings of SRF2009, Berlin, Germany, 2009.

18. "A Family of L-Band SRF Cavities for High Power Proton Driver Applications", R.A. Rimmer, F. Marhauser, Proceedings of PAC09, Vancouver, BC, Canada 2009.

19. "Fundamental Power Coupler Development for the SNS Superconducting Linac Cavities", M. Stirbet et. al., Proceedings of LINAC2002, Gyeongju, Korea, 2002.

20. "A High-Power L-Band RF Window", R.A. Rimmer et. al., Proc. PAC 2001, Chicago., LBNL-47968, LAUR 01-2574.

21. "The Jlab Ampere-Class Cryomodule Conceptual Design", R.A. Rimmer et. al., Proceedings of EPAC2006, Edinburgh, Scotland. 2006

22. "Assembly Preparations for the International ERL Cryomodule at Daresbury Laboratory", P. A. McIntosh et. al., Proceedings of SRF2009, Berlin, Germany 2009. 